\section{P04.02 A NOVEL CANCER IMMUNOTHERAPY COMBINES RMVA-CD40L WITH TUMOR TARGETING ANTIBODIES}

${ }^{1} \mathrm{M}$ Hinterberger*, ${ }^{1} \mathrm{~J}$ Medina-Echeverz, ${ }^{1} \mathrm{M}$ Testori, ${ }^{1} \mathrm{M}$ Geiger, ${ }^{1} \mathrm{R}$ Giessel, ${ }^{1} \mathrm{~B}$ Bathke, ${ }^{1} \mathrm{R}$ Kassub, ${ }^{1} \mathrm{~F}$ Gräbnitz, ${ }^{1} \mathrm{G}$ Fiore, ${ }^{1} \mathrm{~S}$ Wennier, ${ }^{1} \mathrm{P}$ Chaplin, ${ }^{2} \mathrm{M}$ Suter, ${ }^{1} \mathrm{H}$ Hochrein, ${ }^{1} \mathrm{H}$ Lauterbach. ${ }^{1}$ Bavarian Nordic, Martinsried, Germany; ${ }^{2}$ Universität Zürich, Zürich, Switzerland

\subsection{6/jitc-2020-ITOC7.71}

Background Virus-based vaccines and appropriate costimulation potently enhance antigen-specific $\mathrm{T}$ cell immunity against cancer. In the present study, we exploit both innate and adaptive immune responses triggered by a novel recombinant modified vaccinia virus Ankara (rMVA) encoding a Tumor-Associated Antigen (TAA) and the costimulatory CD40L against solid tumors in combination regimes to overcome tumor-induced resistance to immunotherapy.

Material and Methods Subcutaneous murine tumors were induced in $\mathrm{C} 57 \mathrm{BL} / 6$ or $\mathrm{Balb} / \mathrm{c}$ mice using syngeneic tumor cell lines. When tumors were established $\left(60-80 \mathrm{~mm}^{3}\right)$ mice were intravenously injected with rMVA-CD40L. Tumor growth monitoring and immune cell analysis was performed.

Results Therapeutic treatment with rMVA-CD40L resulted in the control of established tumors in several independent tumor models. This antitumor effect was based on the generation of non-exhausted, systemic tumor-specific cytotoxic $\mathrm{CD}^{+}{ }^{+} \mathrm{T}$ cells that was essential for therapeutic efficacy. Strikingly, rMVA-CD40L also induced strong NK cell activation and enhanced cytotoxicity. Moreover, the combination of rMVA-CD40L and tumor targeting antibodies resulted in increased therapeutic antitumor efficacy. This therapeutic combination relied on $\mathrm{Fc} \gamma$ receptor-expressing immune cells as well as on NK cells.

Conclusion We describe a novel and translationally relevant therapeutic synergy between viral vaccination and CD40L costimulation. We show strengthened antitumor immune responses when both rMVA-CD40L-induced innate and adaptive immune mechanisms are exploited by combining immunotherapeutic regimes, such as TAA targeting antibodies. This finding could have a direct positive impact in therapeutic regimens where TAA targeting antibodies could be employed.

Disclosure Information M. Hinterberger: A. Employment (full or part-time); Significant; Bavarian Nordic. J. Medina-Echeverz: A. Employment (full or part-time); Significant; Bavarian Nordic. M. Testori: A. Employment (full or part-time); Significant; Bavarian Nordic. M. Geiger: A. Employment (full or part-time); Significant; Bavarian Nordic. R. Giessel: A. Employment (full or part-time); Significant; Bavarian Nordic. B. Bathke: A. Employment (full or part-time); Significant; Bavarian Nordic. R. Kassub: A. Employment (full or part-time); Significant; Bavarian Nordic. F. Gräbnitz: A. Employment (full or part-time); Significant; Bavarian Nordic. G. Fiore: A. Employment (full or part-time); Significant; Bavarian Nordic. S. Wennier: A. Employment (full or part-time); Significant; Bavarian Nordic. P. Chaplin: A. Employment (full or part-time); Significant; Bavarian Nordic. M. Suter: A. Employment (full or part-time); Significant; Bavarian Nordic. H. Hochrein: A. Employment (full or part-time); Significant; Bavarian Nordic. H. Lauterbach: A. Employment (full or part-time); Significant; Bavarian Nordic.

\section{P04.03 \\ IMMUNE MODULATORY VACCINE DIRECTED AGAINST ID01-EXPRESSING IMMUNE CELLS ELICITS T CELL- MEDIATED ANTI-TUMOR IMMUNITY AND ENHANCES ANTI-PD1 RESPONSES}

${ }^{1} S$ Dey, ${ }^{1}$ E Sutanto-Ward, ${ }^{2}$ KL Kopp, ${ }^{1} \mathrm{JB}$ DuHadaway, ${ }^{1} \mathrm{~A}$ Mondal, ${ }^{2}$ Lecoq, ${ }^{2} \mathrm{M}$ Zocca, ${ }^{2,3} \mathrm{MH}$ Andersen, ${ }^{2} \mathrm{AW}$ Pedersen*, ${ }^{1} \mathrm{AJ}$ Muller. 'Lankenau Institute for Medical Research, Wynnewood, PA, USA; ${ }^{2} I 0$ Biotech, Copenhagen, Denmark; ${ }^{3}$ Centre for Cancer Immunotherapy, Copenhagen, Denmark

\subsection{6/jitc-2020-ITOC7.72}

Background Indoleamine 2,3-dioxygenase 1 (IDO1) is a tryptophan-catabolizing enzyme that contributes to immunoregulation at many levels, including suppressing effector $\mathrm{T}$ cells and inducing/activating regulatory $\mathrm{T}$ cells. Thus far, several therapeutic approaches to target IDO1 enzymatic activity have shown promise in preclinical models, however, results from the first major clinical trial were disappointing. The present study seeks to provide preclinical PoC data for the conceptually unique idea of developing an IDO1-targeted vaccine based on our earlier findings that humans exhibit intrinsic $\mathrm{T}$ cell reactivity against IDO1 epitopes ${ }^{1}$ suggesting the existence of a $\mathrm{T}$ cell-mediated, counter-regulatory mechanism directed against cells that express IDO1.

Materials and Methods IDO1-derived peptide vaccines were identified by measurement of vaccine-induced ex vivo response (IFN $\gamma$ ELISpot) and demonstration of anti-tumor responses in CT26 tumor-bearing mice. To understand the vaccine's mode of action, resected tumors were analyzed by immunofluorescence microscopy and flow cytometry.

Results The CT26 colon carcinoma model was selected for these studies based on evidence of high levels of IDO1 expression and responsiveness to IDO1 inhibition reported for these tumors. In silico-predicted $\mathrm{H} 2^{\mathrm{d}} \mathrm{MHC}$ class $\mathrm{I}$ and II-restricted IDO1 peptide sequences were tested and vaccine candidates were chosen after confirming ex vivo response and anti-tumor response in CT26. Therapeutic treatment of established CT26 tumors with MHC class I- and II-directed, IDO1-derived peptide vaccines elicited anti-tumor responses when administered alone, and the effect was further pronounced when combined, suggesting distinct mechanisms of action. In addition, a combination of IDO1 vaccine with anti-PD-1 antibody produced a combinatorial anti-tumor response beyond what was achieved with either agent alone. Consistent with this observation, adoptive transfer of isolated CD8 + T cells from class I and CD4 $+\mathrm{T}$ cells from class II peptide-vaccinated responder mice delayed tumor growth in treatment naïve mice. The class II-directed response was completely IDO1-dependent while the class I-directed response included an IDO1-independent component indicative of antigen spread. Examination into the tumors in vaccinated mice indicated that IDO1 vaccine treatment exerts its effect by selective reduction of IDO1 expression in the tumor microenvironment and concomitant expansion of activated $\mathrm{CD}^{+}$and $\mathrm{CD} 8^{+} \mathrm{T}$ cells.

Conclusions As noted in humans, our data demonstrate that IDO1 is immunogenic in mice confirming that this endogenous protein is excluded from normal tolerance mechanisms. The observed immunotherapeutic efficacy of IDO1 peptide vaccines on their own and in combination with anti-PD-1 antibody support the rationale for ongoing clinical development of IDO1 peptide vaccine-based therapy. Future studies include further differentiation of the vaccine platform against 
other IDO1-targeting approaches, as well as decoding the underlying mechanism of cooperativity between anti-PD-1 antibody and IDO1 peptide vaccines.

\section{REFERENCE}

1. Sørensen RB, Hadrup SR, Svane IM, Hjortsø MC, Thor Straten P, Andersen MH. Indoleamine 2,3-dioxygenase specific, cytotoxic $T$ cells as immune regulators. Blood 2011; 117(7): 2200-10

Disclosure Information S. Dey: None. E. Sutanto-Ward: None. K.L. Kopp: A. Employment (full or part-time); Significant; IO Biotech. J.B. DuHadaway: None. A. Mondal: None. I. Lecoq: A. Employment (full or part-time); Significant; IO Biotech. M. Zocca: A. Employment (full or part-time); Significant; IO Biotech. M.H. Andersen: A. Employment (full or part-time); Significant; IO Biotech. A.W. Pedersen: A. Employment (full or part-time); Significant; IO Biotech. A.J. Muller: F. Consultant/ Advisory Board; Modest; IO Biotech.

\section{P04.04 MULTIFUNCTIONAL ANTIBODY CONSTRUCT FOR IN VIVO TARGETING OF DENDRITIC CELLS AS A THERAPEUTIC VACCINATION STRATEGY IN AML}

\begin{abstract}
${ }^{1} \mathrm{~S}$ Schmitt*, ${ }^{2,3} \mathrm{~A}$ Lohner, ${ }^{2,3} \mathrm{~K}$ Deiser, ${ }^{4} \mathrm{~A}$ Maiser, ${ }^{2,3} \mathrm{M}$ Rothe, ${ }^{2,3} \mathrm{C}$ Augsberger, ${ }^{5} \mathrm{~A}$ Moosmann, ${ }^{4} \mathrm{H}$ Leonhardt, ${ }^{1} \mathrm{~N}$ Fenn, ${ }^{6} \mathrm{M}$ Griffioen, ${ }^{1} \mathrm{~K}$ Hopfner, ${ }^{2,3,7} \mathrm{M}$ Subklewe. ${ }^{1}$ Department of Biochemistry, Gene Center of the LMU Munich, Munich, Germany; ${ }^{2}$ Department of Medicine III, University Hospital, LMU Munich, Munich, Germany; ${ }^{3}$ Laboratory for Translational Cancer Immunology, Gene Center of the LMU Munich, Munich, Germany; ${ }^{4}$ Department of Biology II, Center for Integrated Protein Science Munich, Munich, Germany; ${ }^{5}$ Research Unit Gene Vectors, Haematologikum, Helmholtz Center Munich, Munich, Germany; ${ }^{6}$ Department of Hematology, Leiden University Medical Center, Leiden, Netherlands; ${ }^{7}$ German Cancer Consortium (DKTK) and German Cancer Research Center (DKFZ), Heidelberg, Germany
\end{abstract}

\subsection{6/jitc-2020-ITOC7.73}

Background Dendritic cells (DCs) are antigen-presenting cells that induce antigen-specific T-cell responses. Therefore, they are used as tools and targets for anti-tumor vaccination. In contrast to T-cell based immunotherapies, that are often limited to surface antigens, DC-based vaccination strategies open up new therapeutic options by utilizing highly abundant intracellular tumor antigens as a target source. Among those, recent interest has been focused on the identification of neoantigens derived from tumor-specific mutations. Especially mutated Nucleophosmin 1 ( $\triangle \mathrm{NPM} 1)$ is a considered candidate for targeted therapy in acute myeloid leukemia (AML). We developed a multifunctional antibody construct consisting of a peptide domain including a variable T-cell epitope that is fused to an $\alpha \mathrm{CD} 40$ single chain variable fragment (scFv) with agonistic function to target and activate dendritic cells in vivo. To potentiate therapeutic efficacy, toll-like receptor (TLR) agonists can be attached as co-stimulatory domains, thereby aiming to enhance cross-presentation of conjugated (neo)antigens to CD8 + T cells.

Materials and Methods Flow cytometry and microscopy-based binding and internalization experiments were performed using monocyte-derived dendritic cells (moDCs). Upregulation of surface markers (CD80, CD83, CD86, HLA-DR) as well as cytokine secretion (IL-6 and IL-12) indicated DC maturation. To validate peptide processing and presentation, moDCs were co-cultured with autologous as well as allogeneic $\mathrm{T}$ cells. IFN- $\gamma$ and TNF- $\alpha$ secretion served as a readout for Tcell activation, peptide-MHC multimer staining for T-cell proliferation.
Results For proof-of-principle experiments, the multispecific antibody derivative was developed by fusing the $\alpha \mathrm{CD} 40 \mathrm{scFv}$ to a cytomegalovirus (CMV)-specific peptide. The $\alpha \mathrm{CD} 40$. CMV construct bound CD40 agonistically and showed efficient internalization into early endosomal compartments on immature moDCs. In co-cultures of immature and mature moDCs with autologous or allogeneic T cells, $\alpha$ CD40.CMV induced a significantly increased T-cell activation and proliferation compared to the control. The co-administration of $\alpha C D 40 . C M V$ with various TLR agonists as vaccine adjuvants resulted in a significant upregulation of DC maturation markers in comparison to $\alpha \mathrm{CD} 40 . \mathrm{CMV}$ only. Interestingly, not all adjuvants were able to enhance the T-cell response. To translate this principle to the AML setting, the CMV peptide sequence was replaced with the $\triangle$ NPM1-derived and HLAA*02:01-binding neoantigen CLAVEEVSL. Cross-presentation to $\mathrm{CD} 8+\mathrm{T}$ cells transduced with a $\Delta$ NPM1-specific T-cell receptor was proven by IFN- $\gamma$ and TNF- $\alpha$ secretion in co-cultures with moDCs that have been pre-incubated with $\alpha C D 40 . \triangle N P M 1$. The optimal vaccine adjuvant has yet to be identified.

Conclusions We successfully demonstrated the development of a multifunctional antibody construct that specifically targets and stimulates DCs by an agonistic $\alpha C D 40 \mathrm{scFv}$. It simultaneously delivers a $\mathrm{T}$ cell-specific peptide with a vaccine adjuvant to induce an efficient T-cell response. As neoantigens are promising targets and under intense investigaton, the $\alpha C D 40 . \triangle N P M 1$ fusion protein is of high therapeutic interest. Thus, our approach displays a promising DC vaccination option for the treatment of AML.

Disclosure Information S. Schmitt: None. A. Lohner: None. K. Deiser: None. A. Maiser: None. M. Rothe: None. C. Augsberger: None. A. Moosmann: None. H. Leonhardt: None. N. Fenn: None. M. Griffioen: None. K. Hopfner: None. M. Subklewe: None.

\section{P04.05 MODULATING TUMOR MICROENVIRONMENT WITH ARGINASE-1 SPECIFIC T CELLS}

${ }^{1} \mathrm{E}$ Martinenaite* ${ }^{2} \mathrm{M}$ Aaboe Joergensen, ${ }^{2} \mathrm{RE}$ Johansson Mortensen, ${ }^{2} \mathrm{~S}$ Munir Ahmad, ${ }^{2}$ SE Weis-Banke, ${ }^{2} \mathrm{M}$ Orebo Holmström, ${ }^{1} \mathrm{~A}$ Wakatsuki Pedersen, ${ }^{2} \mathrm{O}$ Met, ${ }^{2} \mathrm{IM}$ Svane, ${ }^{2} \mathrm{M}$ Hald Andersen. ${ }^{1} \mathrm{O} O$ Biotech, Copenhagen, Denmark; ${ }^{2}$ National Center for Cancer Immune Therapy, Department of Oncology, Copenhagen University Hospital, Herlev, Denmark

\subsection{6/jitc-2020-ITOC7.74}

Background Cancer progression is associated with an increased immune suppression at the tumor site. Arginase- 1 is an enzyme well-known for its involvement in metabolic immune regulation. At the tumor site, arginase- 1 acts by reducing availability of Larginine to the infiltrating immune cells thus reducing $\mathrm{T}$ cell functionality and proliferation. While arginase- 1 is expressed by some tumor cells, it has also been shown to be produced by immune inhibitory myeloid cells, such as myeloid derived suppressor cells (MDSCs), tumor associated macrophages (TAMs) and is associated with poor prognosis. Previously, we demonstrated that spontaneous $\mathrm{CD} 4+$ and $\mathrm{CD} 8+$ T-cell immune responses against arginase-derived, HLA-restricted peptides can be found in both cancer patients and healthy individuals (Martinenaite et al, 2018, DOI: 10.1080/2162402X.2017.1404215). These $\mathrm{T}$ cells are present in the memory $\mathrm{T}$ cell compartment, and that they are activated in arginase- 1 inducing conditions, 